\title{
Prevalence of Pneumothorax with Respect to Type, Etiology, Severity, Extent, Anatomical Location, and Mode of Presentation in Bhuj Population, India
}

\author{
Dr. Dinesh Dave ${ }^{1}$, Dr. Nishant Pujara ${ }^{2}$ \\ ${ }^{1,2}$ Assistant Professor, Department of Medicine, Gujarat Adani Institute of Medical Science, Bhuj, India
}

\begin{abstract}
Pneumothorax may be spontaneous or traumatic. The spontaneous pneumothorax is usually the result of rupture of superficial emphysematous bullae and may occur in apparently healthy persons or in association with chronic pulmonary disease. It is usually unilateral but may be bilateral. If air reaches the mediastinum and rupture into both the pleural cavity. 25 consecutive cases of pneumothorax admitted at the Gujarat Adani institute of medical science bhuj from December 2011 to April 2012were studied. Each patient was interrogated and examined in detail according to the attached proforma, with a view to identify the nature of pneumothorax. The incidence of pneumothorax was maximum in the $3^{\text {rd }}$ decade of life. The youngest one was a boy ages 17 years, while the oldest case was 70 years. 18 cases were under the age of 40 years, while only 1 case was more than 70 years of age. The prevalence of pneumothorax was greater in male as compared to female. Most of the cases treated with needle aspiration of air and the intercostal drainage through indwelling polythelene catheter developed surgical emphysema on the affected side which disappeared within 2-3 days after aspiration if air with needle or after removal of polythelene tube from intercostal space.
\end{abstract}

Keywords: Pneumothorax, Etiology, Type, Bhuj

\section{Introduction}

Pneumothorax may be defined as presence of air in the pleural cavity. The pleural space normally contains no air and only few millimeters of lubricating fluid. When air enters the pleural space, the lung collapses in proportion to the amount of air present. It may be so little as to escape detection of so massive as to threaten life as in tension pneumothorax. Prompt recognition of tension pneumothorax is of utmost importance and it should be treated quickly. ${ }^{1}$

Pneumothorax may be spontaneous or traumatic. The spontaneous pneumothorax is usually the result of rupture of superficial emphysematous bullae and may occur in apparently healthy persons or in association with chronic pulmonary disease. It is usually unilateral but may be bilateral. If air reaches the mediastinum and rupture into both the pleural cavity. ${ }^{2}$

Spontaneous pneumothorax is easily diagnosed clinically but on rare occasion it is diagnosed accidently on routine $\mathrm{x}-$ ray examination.

In 1915 studies of Fischer and Haynashi emphasized the significance of rupture emphysematous bullae in development of pneumothorax. In 1931 Kjaergard emphasized the role of subpleural apical blebe leading to a rupture and development of pneumothorax in majority of patients. Perry in 1939 stated that mild case requires no special treatment and rest in bed to one week should be sufficient. $^{3}$

In the present series, a detailed study of 25 cases of spontaneous pneumothorax was undertaken with type, etiology, severity, extent, anatomical location, and mode of presentation, clinical features, recurrences, complications and result of treatment.

\section{Material \& Methods}

25 consecutive cases of pneumothorax admitted at the Gujarat Adani institute of medical science bhuj from December 2011 to April 2012 were studied. Each patient was interrogated and examined in detail according to the attached proforma, with a view to identify the nature of pneumothorax, its etiology, type, severity, extent, anatomical location, mode of presentation, clinical features, complications and the results of treatment.

Each subject was subjected to intrapleural pressure study to determine the type of pneumothorax i.e. closed, open or tension pneumothorax by artificial pneumothorax apparatus as follows:-

\section{Results}

The incidence of pneumothorax was maximum in the $3^{\text {rd }}$ decade of life. The youngest one was a boy ages 17 years, while the oldest case was 70 years. 18 cases were under the age of 40 years, while only 1 case was more than 70 years of age. The prevalence of pneumothorax was greater in male as compared to female.

The incidence of pneumothorax was higher in person with sedentary occupation as compared to the manual worker, but since the series is small and so also the difference no definite conclusion can be drawn. In majority cases of pneumothorax, paroxysm of cough was the precipitating factor of spontaneous pneumothorax. Lifting heavy weights precipitated pneumothorax in 1 case. While in 9 cases there were no apparent precipitating factors.

History of heavy smoking i.e. 25 to 50 bodies per day for more than 20 to 25 years was present in 13 cases, 2 cases were in habit of smoking occasionally for at least for 5 years, 10 cases were nonsmokers. Most of the cases 


\section{International Journal of Science and Research (IJSR) \\ ISSN (Online): 2319-7064}

Index Copernicus Value (2013): 6.14 | Impact Factor (2014): 5.611

complained of dyspnea, chest pain, cough and restlessness. In present series, pneumothorax was more common on left side of chest. Mediastinum shift was present in 18 cases. Pulmonary tuberculosis was the etiological factor in 12 cases. Whereas chronic bronchitis with emphysema was present in 11 cases. In 1 case subpleural bullae was the underlying cause. In 1 case no underlying cause was apparent.

16 cases had a closed type of pneumothorax, whereas 8 cases had open pneumothorax, I case had tension pneumothorax.

\section{Discussion}

In present study the cases of spontaneous pneumothorax reported for treatment with duration of illness ranging from 2 days to two and half months. 4 cases of pneumothorax gave history suggestive of pneumothorax more than month. These 4 cases had bronchial asthma, chronic bronchitis with emphysema and pulmonary tuberculosis as the underlying etiology of pneumothorax. 20 cases had duration of illness less than 1 month. Out of these 20 cases, 8 cases had duration of illness less than 1 week. All these cases expect the female cases and cases falling in the age group of 11 20 years were in the habit of smoking.

The highest incidences of spontaneous pneumothorax in the present series were found in age of $21-30$ years which tallies with observation of William Bernhard. Next to this age group the incidence of spontaneous pneumothorax was common in age group of $31-40$ years.

In short, spontaneous pneumothorax is commonly forced in $2^{\text {nd }}, 3^{\text {rd }}, 4^{\text {th }}$ decade of life. Higher incidence is found in this age group is due to the effect of mechanical stresses on less compliant lung. Mechanical stress would affect the apices of the lung. Most high apical stresses are associated with large alveoli at the apex of lung and more negative intrapleural pressure at the apex than elsewhere in lungs. ${ }^{5}$

From the above study it is obvious that incidence of pneumothorax is exceedingly common in male as compared to the female in present series as also in the series reported by other authors. In present series, 24 cases were males and only 1 case female. Males are more exposed to mechanical stresses due to strenuous work. Their smoking habit may also make them more prone to the production of chronic bronchitis and emphysematous changes in lungs.

In the present series in 15 cases, the precipitating factor for spontaneous pneumothorax was paroxysms of cough while it is not report as a precipitating factor by Lercy Hyde and Wiliam Smith and only in $1 \%$ cases in the study of reported by Levy. This gross difference in the incidence of paroxysm cough as precipitating factor in the present study and those reported by other authors is difficult to explain. Rarely pneumothorax is precipitated on rising from bed in early morning. Decreased in ventilation and inactivity during sleep allows retention od secretions in the terminal bronchioles leading to local atelectasis with over distention of adjacent areas of early emphysematous lung, terminating in rupture of bleb. ${ }^{6}$
The results shows that dyspnea and chest pain were commonest symptoms, in cases of pneumothorax in all except William Bernhald series in which dyspnea was present in only $26 \%$ of cases. Symptoms of dyspnea are produced when collapse of lung is more than $40 \%$. Dyspnea is caused by massive collapse of lung aggravated by associated chronic respiratory diseases. Chest pain is due to partial pleura by the air aggravated by pleural inflammation and adhesions. ${ }^{7}$

In the present the commonest etiology of spontaneous pneumothorax was tuberculosis 12 cases, while in wiliam's series it account for $20 \%$ of the cases. Present study reveals that pulmonary tuberculosis stills account for nearly half the cases of spontaneous pneumothorax, unlike the series reported by western authors probably due to the higher incidence of pulmonary tuberculosis in India.

In present series, second common cause of spontaneous pneumothorax was chronic bronchitis with emphysema, whereas in series reported by William, it accounted for $18.2 \%$ of cases, in Levy series $30.2 \%$ of cases and William smith series $32 \%$ of cases. ${ }^{8}$

The observation in the present series showed that spontaneous pneumothorax occurred more on left side while in other series incidence was more common on right side. In the present series only $32 \%$ cases had spontaneous pneumothorax on the right side.

In the present series common complication of pneumothorax was emphysema in 2 cases and hydropneumothorax in 2 cases. Levy in his series did not came across empyema in any of their cases. However hydropneumothorax was reported in $4.5 \%$ in cases reported by Laroy. ${ }^{9}$ Recurrences of pneumothorax were reported in $27 \%$ and $18.5 \%$ of cases in the series reported by Leroy and Levy respectively, while there was not a single case recurrence in the present series.

\section{Treatment}

In the present series 5 cases of pneumothorax were treated with conservative treatment. In these cases collapses of lung was less than $20 \%$ and were treated with complete bed rest, analgesics, antibiotics to prevent pleural infection, bronchodilator drugs in cases where chronic bronchitis with emphysema was the underlying etiology. All of them showed complete re expansion of lung within 3 weeks without aspiration of air.

In 10 cases, where collapse of the lung was less than $30 \%$ but more than $20 \%$ were treated with needle aspiration of air on one or more occasion with complete reexpansion of lung in two weeks' time. In 10 cases of pneumothorax where the collapse of lung was more than $30 \%$, were treated with insertion on intercostal polythelene tube in the second intercostal space and connected with under water seal drainage bottle. Most of the cases treated with needle aspiration of air and the intercostal drainage through indwelling polythelene catheter developed surgical emphysema on the affected side which disappeared within 23 days after aspiration if air with needle or after removal of polythelene tube from intercostal space.

\section{Volume 4 Issue 12, December 2015}




\section{International Journal of Science and Research (IJSR) \\ ISSN (Online): 2319-7064}

Index Copernicus Value (2013): 6.14 | Impact Factor (2014): 5.611

In present series, 13 cases having pulmonary tuberculosis as a underlying etiology of spontaneous pneumothorax were treated with antituberculous drugs.

\section{References}

[1] Wilson MM: Gas Embolism Syndromes: Venous Gas Emboli, Arterial Gas Emboli, and Decompression Sickness.

[2] Winer-Muram HT, Rubin SA: Thoracic complications of tuberculosis. Journal of thoracic imaging 1990, 5:46-63.

[3] Hughes F, Kraeft N, Lowry C: Treatment of idiopathic spontaneous pneumothorax. Journal of the American Medical Association 1951, 146:244-7.

[4] Pryles CV: STAPHYLOCOCCAL PNEUMONIA IN INFANCY AND CHILDHOOD An Analysis of 24 Cases. Pediatrics 1958, 21:609-23.

[5] West J: Distribution of mechanical stress in the lung, a possible factor in localisation of pulmonary disease. The Lancet 1971, 297:839-41.

[6] Hough A: Physiotherapy in respiratory care: an evidencebased approach to respiratory and cardiac management: Nelson Thornes, 2001.

[7] Bernhard WF, Malcolm JA, Berry RW, Wylie RH: A study of the pathogenesis and management of spontaneous pneumothorax. CHEST Journal 1962, 42:403-12.

[8] Wagner JA, Langenfeld H, Klett L, Störk S: Activated protein $\mathrm{C}$ in patients with septic shock: a consecutive case series. International journal of clinical pharmacy 2012, 34:23-6.

[9] HYDE L: Benign spontaneous pneumothorax. Annals of internal medicine 1962, 56:746-51. 